\title{
Efeitos de um Procedimento de Ensino de Produção de Seqüências por Sobreposição sob Controle Condicional em Crianças Surdas
}

\author{
Ruth Daisy Capistrano de Souza \\ Grauben José Alves de Assis \\ Priscila Giselli Silva Magalhães \\ Universidade Federal do Pará \\ Paulo Sérgio Teixeira Prado \\ Universidade Estadual Paulista "Júlio de Mesquita Filho", Campus de Marília
}

\begin{abstract}
RESUMO
No presente estudo, cinco crianças surdas foram ensinadas a produzir seqüências de estímulos pelo procedimento de sobreposição sob controle condicional. O procedimento era informatizado. Os estímulos eram apresentados na tela do computador e o participante devia selecioná-los seqüencialmente (por exemplo: A1 $\rightarrow$ A2, na presença da cor verde e $\mathrm{A} 2 \rightarrow \mathrm{A} 1$, na presença da cor vermelha). Uma seqüência correta produzia uma animação gráfica contingentemente à resposta e elogios em Língua Brasileira de Sinais. Foram ensinadas quatro seqüências de dois termos. Seqüências diferentes daquelas programadas como corretas produziam o escurecimento da tela por $3 \mathrm{~s}$ e a reapresentação da tentativa. $\mathrm{O}$ critério de acerto adotado exigiu três tentativas consecutivas sem erro. Todos os participantes alcançaram o critério de acerto, um precisou de re-exposição às condições de ensino. Em seguida, testes foram aplicados. Nos testes de transitividade (p. ex. A1 $\rightarrow$ A3), conectividade (p. ex. $\mathrm{A} 1 \rightarrow \mathrm{B} 2 \rightarrow \mathrm{A} 3$ ), transferência de funções (p. ex. A1 $\rightarrow \mathrm{D} 2$ ) e de generalização (p. ex. uma borracha, dois copos e três caixas), todos os participantes responderam conforme o programado na maioria das tentativas. Os resultados corroboraram a eficiência do procedimento, sugerindo que a equivalência de estímulos seqüenciais pode ocorrer mesmo sob controle condicional em surdos. Pretende-se ampliar esse estudo para contingências de cinco termos.
\end{abstract}

Palavras-chave: produção de seqüências; classes ordinais; relações ordinais; relações de equivalência; discriminação condicional.

\section{ABSTRACT \\ Effects of a Teaching by Overllaping Two-Stimulus Under Conditional Control Procedure in deaf Children}

In the present study, five deaf children were taught to produce stimuli sequences by an overlapping procedure under conditional control. The procedure was computerized. The stimuli were presented on the screen of the computer and the participants were to select them sequentially (e.g., A1 $\rightarrow \mathrm{A} 2$, in the presence of the green color and $\mathrm{A} 2 \rightarrow \mathrm{A} 1$ in the presence of the red color). If the sequence was produced in agreement with the programmed contingencies, an animation was presented for the correct answer plus praise signs in Brazilian Language of Signs. Different sequences produced the darkening of the screen for $3 \mathrm{~s}$ and the re-presentation of the trial. The criterion of performance required three consecutive attempts without errors. All participants performed the criterion, one of them needed re-exposition to the teaching conditions. Next, behavioral tests were applied. In the transitivity (e.g., A1 $\rightarrow$ A3), connectivity (e.g.,A $\rightarrow$ B2 $\rightarrow$ A3), transfer of functions (e.g., A1 $\rightarrow$ D2) and generalization tests (e.g., a rubber, two glasses, three boxes) all participants answered according to the programmed contingencies in most of the trials. The results corroborated the efficiency of the procedure, suggesting that equivalence of sequential stimuli can occur even under conditional control among deaf children. It is our purpose to expand this study to five terms contingencies.

Keywords: sequence production; ordinal classes; ordinal relations; equivalence relations; conditional discrimination. 
Segundo Carmo (2002), "relações ordinais implicam na comparação entre duas situações não equivalentes, sejam estas cardinais, ordinais, de medida ou de seqüência. Nestas situações, um elemento precede ou sucede a outro." (p. 187). O termo ordenação vem sendo usado para designar um tipo de responder seqüencial na presença de um conjunto de estímulos apresentados simultaneamente (Assis, 1987; Assis \& Costa, 2004; Galy, Camps \& Melan, 2003; Mackay \& Brown, 1971; Sampaio \& Assis, 2005; Sidman \& Rosenberger, 1967; Sigurdardottir, Geen \& Saunders, 1990). A análise de desempenhos ordinais emergentes é importante para a compreensão de seqüências complexas, como organização de frases e sentenças (Ribeiro, Assis \& Enumo, 2005). Além disso, como destacam estes mesmos autores:

Pode-se examinar a emergência de classes de equivalência ou a emergência de classes ordinais, pois ambas envolvem treino discriminativo, seguido por transferência de funções de controle de estímulo. Do ponto de vista teórico-metodológico, a formação de classes de equivalência vem sendo avaliada nos estudos sobre formação de conceitos (semântica). A análise de desempenhos emergentes, por sua vez, favorece a compreensão de seqüências complexas, como a organização de frases e sentenças (sintaxe), além de compor as relações entre estímulos e estímulos e respostas envolvidas no comportamento matemático. (p. 123)

\section{Propriedades de uma relação ordinal}

A descrição de relações ordinais baseia-se numa definição matemática para analisar relações estímuloestímulo produzidas pelo ensino de seqüências. De acordo com Green e cols. (1993), as propriedades de uma relação ordinal são: irreflexividade, segundo a qual não é possível um elemento de uma seqüência ser seguido por ele mesmo, em função de sua posição ordinal (logo, A1 $\rightarrow$ A1 (1) não existe); assimetria, cuja característica é ser unidirecional (por exemplo: se $\mathrm{A} 2 \rightarrow \mathrm{A} 3$, então, A3 $\rightarrow$ A2 não é possível nesta mesma seqüência); transitividade, quando $\mathrm{A} 2 \rightarrow \mathrm{A} 3$ e $\mathrm{A} 3 \rightarrow \mathrm{A} 4$, então $\mathrm{A} 2 \rightarrow \mathrm{A} 4$, sendo que, a inferência desta propriedade só pode ser feita com base numa série treinada com pares de estímulos não-adjacentes; conectividade, neste tipo de relação, todos os pares de estímulos estão relacionados entre si (por exemplo: se $\mathrm{A} 1 \rightarrow \mathrm{A} 2 \rightarrow \mathrm{A} 3$, então $\mathrm{A} 1 \rightarrow \mathrm{A} 2, \mathrm{~A} 1 \rightarrow \mathrm{A} 3$ e $\mathrm{A} 2 \rightarrow \mathrm{A} 3)$. É importante destacar que esta propriedade é inferida se todos os pares possíveis (adjacentes e não-adjacentes) de elementos dentro da seqüência forem arranjados em uma ordem consistente.
Segundo Lima e Assis (2003),

A verificação de classes ordinais pressupõe que todos os estímulos na classe sejam mutuamente substituíveis no controle de um mesmo desempenho e que qualquer propriedade controladora adquirida por um membro da classe deva ser compartilhada por todos os outros membros. (p. 82)

$\mathrm{O}$ ensino de seqüências em separado e os efeitos desse ensino na formação de classes ordinais e o que descreve essa classe é que seus elementos sejam mutuamente substituíveis pela sua função ordinal, tem sido estudado por muitos autores a exemplo de Stromer e Mackay (1993).

\section{0 ensino de seqüências por sobreposição de pares de estímulos}

Um procedimento que tem sido empregado em estudos sobre produção de seqüências é conhecido como ensino por sobreposição de estímulos. Os estímulos a serem ordenados são apresentados aos pares. Quando os estímulos de um par estiverem sendo ordenados na seqüência prevista, um novo estímulo é adicionado em substituição a um dos anteriores. Assim, o segundo elemento de uma seqüência passa a ser o primeiro na nova seqüência. Dessa maneira, os elementos que formam a seqüência completa nunca aparecem todos juntos. Por exemplo, para o ensino da seqüência $\mathrm{A} 1 \rightarrow \mathrm{A} 2 \rightarrow \mathrm{A} 3 \rightarrow \mathrm{A} 4 \rightarrow \mathrm{A} 5$, ensina-se inicialmente a ordenação $\mathrm{A} 1 \rightarrow \mathrm{A} 2$, depois $\mathrm{A} 2 \rightarrow \mathrm{A} 3$ e assim por diante (Stromer \& Mackay, 1993).

Mackay, Kotlarchyk e Stromer (1997) ensinaram uma criança de 10 anos com lesão cerebral a discriminar um conjunto de dígitos e palavras correspondentes. Enquanto o professor ditava a palavra, por exemplo, "Two", o participante era ensinado a construir a palavra seqüencialmente (ensino por anagrama) com as letras " $T$ ", "W", "O" apresentadas na tela de um computador. Após cada tentativa, as letras mudavam de posição na tela. Em seguida, as discriminações de outras palavras foram ensinadas gradativamente em seqüência, até completar nove palavras, todas correspondentes aos dígitos de 1 a 9. Os autores ainda aplicaram um teste de nomeação oral (o professor apresentava um dígito ou uma palavra correspondente e perguntava: "O que é isso?"). Em seguida, o participante era submetido a uma avaliação oral em que precisava responder seqüencialmente do menor para o maior, aos números que apareciam na tela. Embora ele tenha falado em voz alta todos os dígitos, os autores 
concluíram que ele não foi capaz de ordená-los apropriadamente.

Esse estudo permitiu a ampliação do repertório de emparelhamento de acordo com o modelo, baseado em uma rede de relações e de nomeação de numerais, palavras escritas e seqüência oral de "zero" a "nove". Um ensino de seqüenciação de numerais impressos (de 0 a 3 ) foi realizado no segundo estudo, ampliando as classes. Os desempenhos emergentes foram: seqüenciação de palavras e numerais cardinais escritos. $O$ procedimento de ensino permitiu uma integração de novos desempenhos ao repertório do participante. Esses resultados corroboram a hipótese de que o ensino de uma seqüência com um estímulo de cada classe de equivalência pode levar os demais estímulos de cada classe a adquirirem a mesma função ordinal.

Em um estudo conduzido por Stromer e Mackay (1993), duas crianças e cinco adultos típicos foram ensinados a compor duas seqüências com cinco estímulos diferentes na seguinte ordem: $\mathrm{A} 1 \rightarrow \mathrm{A} 2 \rightarrow \mathrm{A} 3 \rightarrow \mathrm{A} 4$ $\rightarrow \mathrm{A} 5$, e $\mathrm{C} 1 \rightarrow \mathrm{C} 2 \rightarrow \mathrm{C} 3 \rightarrow \mathrm{C} 4 \rightarrow \mathrm{C} 5$ independentemente da localização inicial dos estímulos na tela do computador. No ensino por sobreposição de estímulos (utilizando um procedimento de fading in para indicar qual estímulo deveria ser tocado primeiro na tela do computador), os estímulos (formas abstratas) eram apresentados aos pares, lado a lado, e à medida que um novo estímulo era adicionado na formação de uma nova seqüência, o primeiro elemento da seqüência anterior era removido, de maneira que todos os elementos que formam a seqüência completa nunca apareciam juntos. Por exemplo, na seqüência $\mathrm{A} 1 \rightarrow \mathrm{A} 2$, a próxima apresentação era $\mathrm{A} 2 \rightarrow \mathrm{A} 3$.

$\mathrm{O}$ ensino envolveu quatro classes ordinais com pares de estímulos sobrepostos (overllaping two-stimulus): p. ex. A1 $\rightarrow \mathrm{A} 2, \mathrm{~A} 2 \rightarrow \mathrm{A} 3, \mathrm{~A} 3 \rightarrow \mathrm{A} 4$ e $\mathrm{A} 4 \rightarrow \mathrm{A} 5$ ou $\mathrm{C} 1 \rightarrow \mathrm{C} 2, \mathrm{C} 2 \rightarrow \mathrm{C} 3, \mathrm{C} 3 \rightarrow \mathrm{C} 4$ e $\mathrm{C} 4 \rightarrow \mathrm{C} 5$.

Testes de transitividade e conectividade foram conduzidos e os resultados indicaram que houve emergência de novas seqüências derivadas com dois estímulos $(\mathrm{C} 2 \rightarrow \mathrm{C} 4)$ com inferência transitiva e com cinco estímulos $(\mathrm{C} 1 \rightarrow \mathrm{A} 2 \rightarrow \mathrm{C} 3 \rightarrow \mathrm{A} 4 \rightarrow \mathrm{C} 5)$ envolvendo permutação de estímulos que assumiram a mesma função ordinal estabelecidas pelo ensino. Concluiu-se que os novos estímulos funcionaram como membros das classes ordinais existentes, ou seja, que foram mutuamente intercambiáveis e que a variação do procedimento de treino com duas classes adjacentes pode fornecer um caminho para isolar e avaliar proprieda- des transitivas potenciais de relações ordinais entre estímulos.

Holcomb, Stromer e Mackay (1997) conduziram um estudo com o objetivo de replicar sistematicamente os resultados obtidos por Stromer e Mackay (1993) e verificar se crianças ensinadas a ordenar pares de estímulos visuais seriam capazes de produzir novas seqüências sem treino adicional. No Experimento 1 , seis crianças com idade entre 3 e 4 anos foram inicialmente ensinadas a produzir uma seqüência de seis formas $(A \rightarrow B \rightarrow C \rightarrow D \rightarrow E \rightarrow F)$. Então, cinco seqüências de dois estímulos sobrepostos (overlaping two-stimuli) foram ensinadas com outro conjunto de formas $(\mathrm{A} 1 \rightarrow \mathrm{A} 2, \mathrm{~A} 2 \rightarrow \mathrm{A} 3, \mathrm{~A} 3 \rightarrow \mathrm{A} 4, \mathrm{~A} 4 \rightarrow \mathrm{A} 5, \mathrm{~A} 5 \rightarrow \mathrm{A} 6$, $\mathrm{B} 1 \rightarrow \mathrm{B} 2, \mathrm{~B} 2 \rightarrow \mathrm{B} 3, \mathrm{~B} 3 \rightarrow \mathrm{B} 4, \mathrm{~B} 4 \rightarrow \mathrm{B} 5, \mathrm{~B} 5 \rightarrow \mathrm{B} 6)$. Todas as crianças foram capazes de ordenar três ou mais estímulos sem treino adicional, expandindo os resultados obtidos por Stromer e Mackay (1993). Contudo, não produziram o desempenho de ordenar com seqüências longas. No Experimento 2, seis outras crianças foram submetidas ao procedimento de sobreposição com cinco seqüências de dois estímulos, no qual o ensino preliminar com uma seqüência de seis estímulos foi eliminado e introduziu-se um procedimento de fading, no qual os estímulos são introduzidos gradativamente, para reduzir o número de respostas diferentes do programado. Todas as crianças aprenderam as seqüências nos treinos e cinco delas produziram, imediatamente após o ensino, seqüências de dois a seis estímulos sem treino direto. O Experimento 3 replicou as mesmas condições do Experimento 2 e incluiu a aplicação de entrevistas após os testes, a fim de avaliar a mediação pelo comportamento verbal nos desempenhos apresentados. Quatro novas crianças participaram do estudo. Os resultados sugerem que a produção de seqüências foi feita sem o recurso a quaisquer estratégias de nomeação pelas crianças. Segundo os autores, a transitividade pode ocorrer mesmo na ausência da mediação verbal e a inferência de que as relações entre os estímulos são transitivas baseia-se no fato de que as seqüências de pares não adjacentes são observadas sem treino direto. Mas o treino estabeleceu pré-requisitos para a demonstração de relações transitivas, pois estes desempenhos foram consistentes com o ensino. A ordem em que os conjuntos de estímulos foram ensinados foi outro fator que, segundo os autores, pode ter facilitado o desempenho, pois se as seqüências sobrepostas fossem ensinadas em uma ordem assistemática, os resultados positivos seriam menos prováveis. 
Mais recentemente, Verdu, Souza e Lopes Jr. (2006) avaliaram a eficácia do procedimento de ensino de pares de relações ordinais compostas por estímulos adjacentes sobre o estabelecimento do comportamento de compor as várias seqüências ensinadas e sobre a emergência de relações ordinais com três, quatro e cinco estímulos. Cinco alunos do primeiro ciclo do ensino básico fundamental com dificuldades de leitura e escrita foram expostos a duas seqüências diferentes com cinco formas abstratas. Estabelecida a linha de base, foram conduzidos testes da emergência de relações ordinais com três, quatro e cinco estímulos dos dois conjuntos A e B, separadamente. Avaliou-se se estímulos das seqüências ensinadas eram mutuamente substituíveis, com base na mesma função ordinal (primeiro, segundo, e assim por diante). Os estudantes compuseram as relações ordinais ensinadas e apresentaram relações ordinais emergentes. Segundo os autores, esses resultados sugerem a formação de cinco classes de estímulos (A1B1, A2B2, e assim por diante), cada uma consistindo de estímulos que ocuparam a mesma função ordinal em diferentes seqüenciais.

As investigações sobre esse fenômeno têm sido realizadas com população com desenvolvimento típico e, daquelas com desenvolvimento atípico sobressaem aquelas com baixo desempenho cognitivo. Somente um estudo (Souza, Assis \& Magalhães, 2005) investigou a emergência de relações ordinais com crianças surdas, usando o procedimento de ensino por sobreposição. Os resultados obtidos demonstraram que os participantes foram capazes de responder a novas seqüências de estímulos não ensinadas diretamente e que o procedimento foi eficiente na aprendizagem de comportamentos conceituais numéricos em crianças surdas. O desempenho emergente destas relações pode ser possível após um ensino por sobreposição de estímulos sob controle condicional com crianças surdas?

Crianças que tiveram contato restrito e limitado com estímulos sonoros seriam capazes de ordenar seqüencialmente sob diferentes condições experimentais? Considerando que a principal característica desses participantes é a privação sensorial auditiva e a aquisição de um repertório verbal espaço-visual como funcionalmente equivalente ao vocal.

Esse foi exatamente o objetivo do presente estudo: verificar os efeitos do procedimento de ensino por sobreposição, sob controle condicional, sobre relações ordinais em crianças com surdez congênita, testar a transferência de funções ordinais e de generalização para novos estímulos do ambiente escolar do participante.

\section{MÉTODO}

\section{Participantes}

Participaram do estudo cinco crianças com idade entre cinco e oito anos. O critério de seleção para o seu recrutamento foi que apresentassem perda auditiva acima de 91 decibéis identificada por meio de exames audiométricos e com laudo emitido por otorrinolaringologista. Segundo alguns autores (Green, 1990), relações condicionais estabelecidas entre conjuntos de estímulos auditivo-visuais foram aprendidas mais rapidamente do que entre conjuntos exclusivamente visuais. Todos eram regularmente matriculados numa unidade de ensino especializado onde freqüentavam uma classe de alfabetização. Dados mais detalhados são apresentados na Tabela 1.

TABELA 1

Relação dos Participantes, Gênero, Idade Cronológica e Perda Auditiva

\begin{tabular}{ccc}
\hline Participante & Gênero & Idade cronológica \\
\hline AMN & $M$ & $7 \mathrm{a} 10 \mathrm{~m}^{*}$ \\
JSO & $\mathrm{F}$ & $6 \mathrm{a}$ \\
PRS & $\mathrm{M}$ & $6 \mathrm{a}$ \\
KVM & $\mathrm{M}$ & $8 \mathrm{a} 10 \mathrm{~m}$ \\
JVG & $\mathrm{M}$ & $7 \mathrm{a} 11 \mathrm{~m}$ \\
\hline
\end{tabular}

${ }^{*} a=$ anos; $m$ = meses.

A participação das crianças no estudo foi autorizada pelos pais ou responsáveis mediante assinatura de um termo de consentimento livre e esclarecido (ver anexo) após reunião na qual um dos autores expôs as linhas gerais e o propósito da pesquisa, bem como esclareceu eventuais dúvidas sobre o projeto. O documento foi redigido nos termos da Resolução 196/96 do Conselho Nacional de Saúde e aprovado pelo Comitê de Ética da Universidade. 


\section{Ambiente experimental}

As sessões experimentais foram conduzidas num compartimento de aproximadamente $2 \mathrm{~m} \times 2 \mathrm{~m} \times 2,10$ $\mathrm{m}$, construído com divisórias de madeira no interior de uma sala mais ampla da escola freqüentada pelos participantes, a qual abriga equipamentos de informática. A parte superior de uma das divisórias é de vidro. À direita da porta de entrada do compartimento ficava uma mesa com o computador utilizado no experimento.

\section{Material e equipamento}

No pré-teste de discriminação de quantidades (ver descrição abaixo) foi utilizado um conjunto de blocos lógicos de madeira, marca FUNBEC. Ele é constituído de 32 peças de diferentes formas (quadrado, triângulo, círculo, retângulo), cores (azul, amarelo, vermelho), tamanhos (grande e pequeno) e espessuras (grosso e fino). Além disso, foram utilizados cartões com desenhos dos sinais em Língua Brasileira de Sinais (LIBRAS) correspondentes aos numerais 1 a 5 e os nomes impressos desses numerais.

Um microcomputador com monitor de 14" foi utilizado para a coleta dos dados. Nas etapas de treino e testes um software não-comercial (REL 4.0 for windows) especialmente desenvolvido para uso em pesquisas, gerenciou a apresentação dos estímulos, o número de tentativas, o posicionamento dos estímulos na tela, bem como efetuou o registro das respostas.

\section{Estímulos}

Foram utilizados quatro conjuntos (ou classes) de estímulos. O conjunto A, composto por desenhos dos sinais, em LIBRAS, correspondentes aos numerais $1 \mathrm{a}$ 5, aqui designados pela notação alfanumérica: A1, A2, A3, A4 e A5; o conjunto B, com uma a cinco formas abstratas (B1 a B5); o conjunto $\mathrm{C}$, formado pelos nomes escritos por extenso dos numerais 1 a $5(\mathrm{C} 1$ a $\mathrm{C} 5)$ e o conjunto $\mathrm{D}$, usado num teste de generalização (Teste de transferência de funções ordinais, descrito posteriormente), também composto por uma a cinco formas abstratas (D1 a D5). Os estímulos descritos são reproduzidos na Figura 1. As cores verde e vermelha serviram como estímulos condicionais. Além disso, num segundo teste de generalização (com estímulos tridimensionais) foram usadas borrachas escolares, copos plásticos e caixas de fósforo.

TABELA 2

Estímulos Utilizados no Estudo

\section{Estímulos}

Classes




\section{Situação}

As sessões foram realizadas numa freqüência de três vezes por semana e tiveram duração de 20 a 30 minutos. Os participantes eram retirados individualmente de sua sala de aulas e conduzidos pela experimentadora ao compartimento anteriormente descrito, sendo reconduzidos à sala de aulas após o término da sessão.

Para a apresentação dos estímulos, a tela do computador foi dividida horizontalmente em duas partes, sendo a parte inferior aqui designada como "área de escolha" e a superior como "área de construção". $\mathrm{Na}$ área de escolha dispunham-se oito quadros de $4,5 \mathrm{~cm}$ $x$ 4,5 cm arranjados em duas fileiras e quatro colunas. Em seu interior, estímulos eram apresentados em posições aleatórias. $\mathrm{Na}$ área de construção havia outros oito quadros de $2,5 \mathrm{~cm} \times 2,5 \mathrm{~cm}$ dispostos em linha (ver Figura 1). Centralizada na parte superior da área de construção, a palavra "TOQUE" era apresentada num quadro com fundo colorido. Um toque com o dedo sobre a palavra produzia a apresentação dos estímulos na área de escolha. Estes, por sua vez, quando tocados deslocavam-se da "área de escolha" para a "área de construção", começando pela primeira "janela" da esquerda para a direita.

\section{PROCEDIMENTO}

Em razão da condição dos participantes, a comunicação entre eles e a experimentadora foi feita por meio da Língua Brasileira de Sinais (LIBRAS). Foram conduzidas avaliações preliminares dos pré-requisitos dos participantes, conforme descrição abaixo:

Discriminação de quantidades. Pré-testes foram aplicados para avaliar a discriminação de quantidades. Para isso foram usados os blocos lógicos, os nomes dos numerais impressos e numerais em LIBRAS. Por exemplo, na presença de um cartão plastificado com o numeral 3 (ou o seu respectivo nome) impresso em LIBRAS, o participante precisava selecionar três blocos lógicos disponíveis sobre a mesa.

Discriminação das cores verde e vermelha. Um teste de nomeação foi aplicado pela experimentadora, em LIBRAS, com as cores (verde e vermelha) que seriam utilizadas no estudo. Peças dos blocos lógicos com cores diferentes eram disponibilizadas sobre a mesa. Conforme os sinais em LIBRAS que indicavam "Qual cor?", apresentados pela experimentadora, o participante deveria apontar a cor correspondente ao sinal verde ou vermelho.
Discriminação de numerosidades. O objetivo do teste foi verificar o controle da resposta pela dimensão de estímulo: numerosidade. A experimentadora retirava de uma caixa contendo todos os blocos lógicos, uma quantidade de peças e perguntava, em LIBRAS: "Quantos têm?". O aluno devia indicar, por meio do sinal apropriado em LIBRAS, o número de peças no bloco lógico. Três tentativas foram programadas. A cada tentativa apresentava-se uma quantidade de peças do bloco lógico de um a seis.

Discriminação das propriedades "muito" e "pouco”. Na presença de dois conjuntos de blocos lógicos com várias peças, apresentavam-se as perguntas, em LIBRAS: "Qual tem muito?" e "Qual tem pouco?". A tarefa do participante era apontar o conjunto com "muitos" ou com "poucos" blocos lógicos.

Relação sinal numérico em LIBRAS-nome impresso correspondente. Neste teste, a experimentadora apresentava, um a um, o sinal em LIBRAS correspondente aos numerais 1 a 5 (ver Tabela 2, Conjunto A) e solicitava ao participante que apontasse o nome impresso do número correspondente (ver Tabela 2, conjunto $\mathrm{C}$ ). Três figuras estavam disponíveis sobre a mesa. Foi previsto um bloco de quinze tentativas, sendo três para cada numeral.

Relação entre desenhos de numerais em LIBRAS e sinais gestuais. Na presença de três desenhos de numerais em LIBRAS (ver Tabela 2, conjunto A), a experimentadora apresentava um sinal gestual. A tarefa do participante era apontar o desenho/numeral correspondente ao sinal gestual apresentado pela experimentadora. Como no teste anterior, houve um bloco de quinze tentativas, três para cada valor de 1 a 5 .

\section{Treino}

O procedimento adotado foi o ensino por sobreposição de pares de estímulos. Duas cores foram introduzidas com a função de estímulos condicionais, de modo que, na presença de uma cor reforçou-se a produção de seqüências de estímulos numa determinada ordem (por exemplo: A1 $\rightarrow \mathrm{A} 2, \mathrm{~A} 2 \rightarrow \mathrm{A} 3$ etc.), ao passo que na presença da outra cor reforçou-se a produção de seqüências de estímulos na ordem inversa (por exemplo: A5 $\rightarrow$ A4, A4 $\rightarrow$ A3 etc.). Iniciou-se o treino com a produção de seqüências de estímulos da classe A. Depois foi conduzido o treino com os estímulos da classe B e, finalmente, com os da classe C. Entre os treinos com cada uma das classes foram introduzidas sondas, testes de transitividade, de conectividade e revisões de linha de base. Detalhes são apresentados a seguir. 
Instruções e seqüência de eventos. Como um recurso para garantir o controle por contingências, optou-se pelo fornecimento de instruções mínimas. Estando o participante ao computador, a experimentadora posicionava-se de modo que ele pudesse ver claramente seus sinais. As instruções eram: "olhe", "preste atenção", "toque na cor". Um toque na janela com fundo colorido centralizada na parte superior da tela produzia a apresentação de pares de figuras na área de escolha, em posições aleatórias. O participante era instruído a tocar numa figura e, em seguida, na outra. A primeira figura tocada se deslocava para o primeiro quadro à esquerda na área de construção. A segunda figura tocada se deslocava para o quadro à direita do primeiro. Uma animação gráfica com duração de $2 \mathrm{~s}$ era apresentada contingentemente à seqüência correta, mais elogios da experimentadora: "muito bem", "certo", "legal". Seqüências incorretas levavam ao escurecimento da tela por $3 \mathrm{~s}$, seguido pela apresentação da mesma configuração de estímulos na área de escolha (procedimento de correção).

Treino e critério de desempenho. O treino foi programado de modo que, na presença da cor verde os estímulos fossem seqüenciados na ordem crescente e, na presença da cor vermelha, na ordem decrescente. Foram exigidos três acertos consecutivos com cada par de estímulos. Assim, três produções da seqüência $\mathrm{A} 1 \rightarrow \mathrm{A} 2$ na presença da cor verde eram necessárias para o início do treino da seqüência inversa. Este, por sua vez, consistia da produção da seqüência A2 $\rightarrow$ A1 na presença da cor vermelha.

Alcançado o critério de desempenho sob essas condições, avançava-se para o treino de produção de seqüência com um novo par de estímulos (A2 $\rightarrow$ A3) na presença da cor verde. Em seguida, treinava-se a ordenação inversa com os estímulos desse novo par na presença da cor vermelha.

O treino seguiu esse padrão até que houvessem sido ensinadas as seqüências A4 $\rightarrow 45$ e A5 $\rightarrow$ A4 na presença das cores verde e vermelha, respectivamente. Concluído o treino com os estímulos da classe A, foram conduzidas sondas e testes de transitividade e conectividade (ver descrição abaixo) e iniciou-se o treino com os estímulos da classe B, também seguido das respectivas sondas e testes. Por fim, procedeu-se da mesma forma com os estímulos da classe $\mathrm{C}$.

Sondas intercaladas. Ao término do treino com os estímulos de cada uma das classes, foram conduzidas sondas com a dupla finalidade de familiarizar os participantes a responderem na ausência de conseqüências - condição semelhante à que seriam expostos nos testes de transitividade e conectividade - e verificar o controle condicional pelas cores. As tentativas de sonda se configuravam da mesma forma que as de treino, com a diferença de não haver qualquer conseqüência programada para as respostas. Duas seqüências corretas com cada par de estímulos foram exigidas: $\mathrm{A} 1 \rightarrow \mathrm{A} 2, \mathrm{~A} 2 \rightarrow \mathrm{A} 3, \mathrm{~A} 3 \rightarrow \mathrm{A} 4$ e A4 $\rightarrow \mathrm{A} 5$ sob a condição "verde" e A5 $\rightarrow \mathrm{A} 4, \mathrm{~A} 4 \rightarrow \mathrm{A} 3, \mathrm{~A} 3 \rightarrow \mathrm{A} 2$ e $\mathrm{A} 2 \rightarrow \mathrm{A} 1$ sob a condição "vermelha", o mesmo se mantendo com relação aos estímulos das classes B e C. Caso o participante respondesse corretamente na primeira tentativa, avançava para o par seguinte.

Testes de transitividade. Para se verificar se as relações ensinadas apresentavam a propriedade de transitividade, testes específicos foram conduzidos. A configuração de estímulos usada foi igual à das tentativas de treino e sonda, com uma diferença fundamental: na área de escolha apresentavam-se pares de estímulos não adjacentes. A transitividade poderia ser inferida se, nessa situação, inédita para o participante, este continuasse a ordenar os estímulos de acordo com os mesmos critérios que orientaram o treino. Também foi avaliada a propriedade sob controle condicional. Assim, na presença da cor verde as seqüências produzidas deveriam ser, por exemplo: $\mathrm{A} 1 \rightarrow \mathrm{A} 3, \mathrm{~A} 2 \rightarrow \mathrm{A} 5$, etc. e, na presença da cor vermelha, elas deveriam ser $\mathrm{A} 4 \rightarrow \mathrm{A} 1, \mathrm{~A} 5 \rightarrow \mathrm{A} 3$, etc. As seqüências de estímulos foram produzidas em extinção. Em todos os testes, caso o participante respondesse diferente do programado, teria mais uma chance com a mesma configuração de estímulos.

Testes de conectividade e teste de formação de classes ordinais. Estes testes foram introduzidos com a finalidade de se verificar se a posição ordinal dos estímulos seria mantida independentemente de eles serem ou não adjacentes ou de pertencerem ou não a uma mesma classe. Adicionalmente, o teste avaliou também se a propriedade se manteria sob controle condicional. Pares de estímulos de duas classes (A e $\mathrm{B}, \mathrm{B}$ e $\mathrm{C}$ ou $\mathrm{A}$ e $\mathrm{C}$, conforme o caso) eram apresentados na área de escolha. Os elementos de cada par eram escolhidos aleatoriamente, porém nunca foram apresentados pares de estímulos de classes distintas que ocupassem uma mesma posição ordinal. Eles deveriam ser seqüenciados em ordem crescente (por exemplo, A1 $\rightarrow \mathrm{B} 2, \mathrm{~B} 4 \rightarrow \mathrm{A} 5$ etc.), na presença da cor verde, e em ordem decrescente na presença da cor vermelha (por exemplo, $\mathrm{C} 2 \rightarrow \mathrm{A} 1, \mathrm{~A} 4 \rightarrow \mathrm{B} 2$ etc.). 


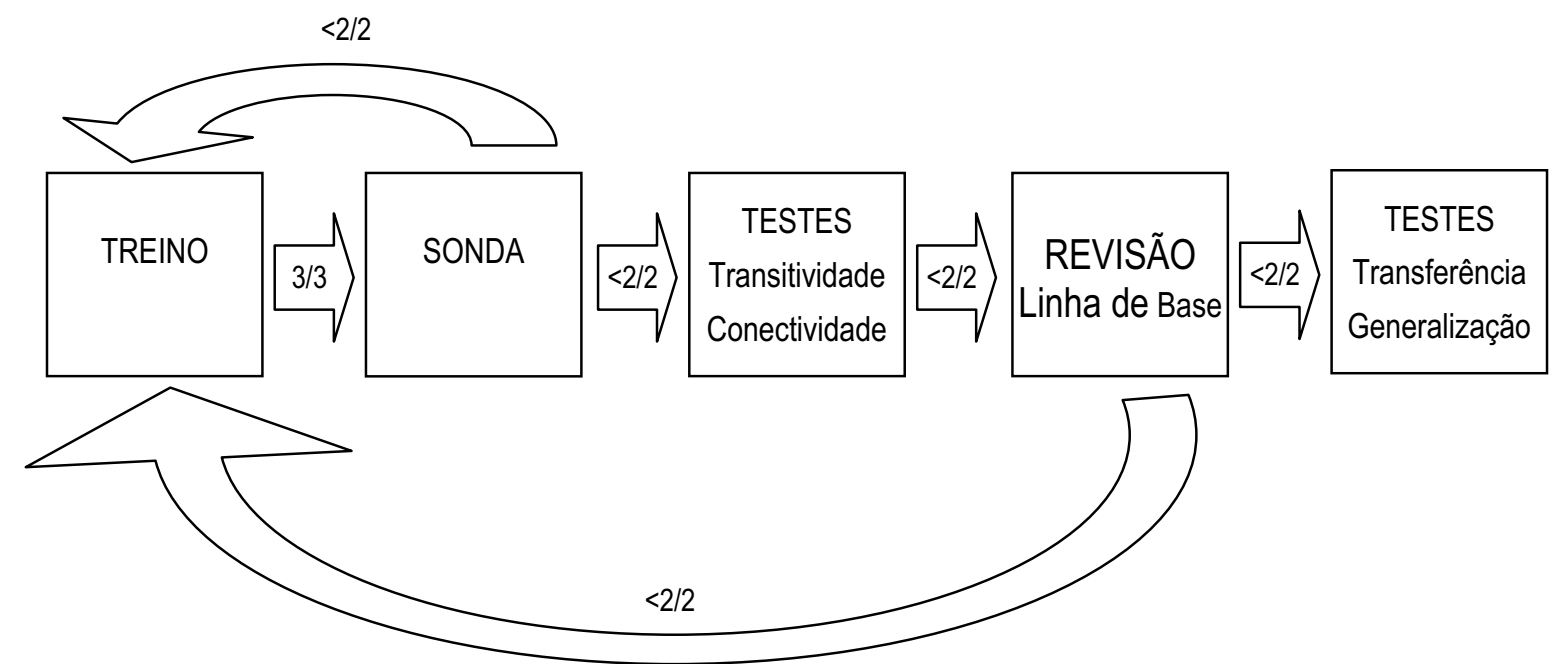

Figura 1. Fluxograma das fases do experimento. 0 critério adotado foi o de três acertos em três tentativas (3/3) para o avanço de uma fase para a seguinte. Desempenho inferior $(<2 / 2)$ levava ao retreino da linha de base. 0 alcance do critério em todas as fases levava ao treino com uma nova classe de estímulos.

Revisões de linha de base. Nesta fase, na presença da cor verde, o participante deveria tocar os pares de estímulos numa ordem crescente. Na presença da cor vermelha, o responder aos pares de estímulos era na ordem inversa. As cores verde e vermelha e seus respectivos pares de estímulos eram apresentados aleatoriamente. Quando o participante respondesse corretamente três vezes consecutivas, um outro par de estímulos era apresentado.

Caso não respondesse corretamente, a tela escurecia por $3 \mathrm{~s}$ e a mesma configuração de estímulos era re-apresentada. As fases do experimento e o critério para o avanço entre elas são resumidos de modo esquemático no fluxograma da Figura 1.

Teste de transferência de funções ordinais. $\mathrm{O}$ objetivo do teste foi verificar se o responder seqüencial se manteria com novos estímulos, isto é, estímulos que não fizeram parte do treino. Foram usados os estímulos da classe D (ver Tabela 2), aos quais o participante deveria responder seqüencialmente sob controle condicional. Não houve conseqüência diferencial para as respostas.

Teste de generalização com estímulos tridimensionais. Neste teste, objetivou-se verificar a generalização do responder seqüencial a estímulos do ambiente natural. Foram usados: cinco borrachas, cinco copos plásticos e cinco caixas de fósforo. Diferentes quantidades de objetos (de um a cinco) foram usadas em cada tentativa. Os objetos estavam disponibilizados sobre uma mesa e solicitava-se ao participante que nomeasse quantos objetos havia sobre a mesa e ordenasse em quantidades de um a cinco.

As tentativas eram conduzidas com os mesmos elementos de um conjunto de objetos ou com elementos diferentes de cada conjuntos de objetos. Não havia conseqüências diferenciais.

\section{RESULTADOS}

No pré-teste, nenhum dos participantes foi capaz de identificar a quantidade de peças disponíveis no bloco lógico, como descrito no procedimento. Todos foram capazes de nomear as cores e identificar o conjunto das peças como "muito" ou "pouco". Nenhum participante foi capaz de relacionar numerais impressos em LIBRAS com os sinais gestuais correspondentes aos numerais.

A Tabela 3 mostra a ordem original de apresentação das tentativas quando todos os participantes alcançaram o critério de acerto, embora alguns precisassem de mais exposições em cada tentativa nos três conjuntos de estímulos A, B e C. Essa ordem de apresentação foi estabelecida para assegurar que o participante discriminasse de fato os aspectos relevantes dos modelos (cores verde ou vermelho) e não alguma outra característica regular eventualmente existente nos estímulos ou no arranjo das tentativas. Assim, uma precaução necessária era assegurar que a ordem de tentativas fosse randomizada pois uma ordem não randomizada permitiria que o participante respondesse à ordem e não aos modelos. Dois participantes (JSO e 
KVM) foram expostos ao procedimento de ensino duas vezes, no máximo. O participante PRS precisou de três exposições na seqüência $\mathrm{C} 2 \rightarrow \mathrm{C} 3$ na presença da cor verde. JVG precisou de três exposições na se- qüência $\mathrm{B} 4 \rightarrow \mathrm{B} 3$ na presença da cor vermelha. O participante $\mathrm{AMN}$ precisou de três ou mais exposições na maioria das tentativas na presença das cores verde e vermelha.

\section{TABELA 3}

Número de Exposições de Cada Participante ao Procedimento de Treino Até o Alcance do Critério

\begin{tabular}{ccccccc}
\hline & & \multicolumn{5}{c}{ Participantes } \\
\cline { 3 - 7 } S $^{*}$ & Tipo de Tentativa & amn & jso & prs & kvm & jvg \\
\hline Verde & $\mathrm{A} 1 \rightarrow \mathrm{A} 2$ & 1 & 1 & 2 & 2 & 1 \\
Vermelho & $\mathrm{A} 2 \rightarrow \mathrm{A} 1$ & 2 & 1 & 2 & 1 & 1 \\
Vermelho & $\mathrm{A} 3 \rightarrow \mathrm{A} 2$ & 1 & 2 & 1 & 1 & 1 \\
Verde & $\mathrm{A} 2 \rightarrow \mathrm{A} 3$ & 1 & 1 & 1 & 2 & 2 \\
Verde & $\mathrm{A} 3 \rightarrow \mathrm{A} 4$ & $>3$ & 1 & 2 & 1 & 1 \\
Vermelho & $\mathrm{A} 4 \rightarrow \mathrm{A} 3$ & 3 & 1 & 2 & 1 & 2 \\
Vermelho & $\mathrm{A} 5 \rightarrow \mathrm{A} 4$ & 1 & 2 & 1 & 2 & 2 \\
Verde & $\mathrm{A} 4 \rightarrow \mathrm{A} 5$ & 3 & 1 & 2 & 2 & 2 \\
Vermelho & $\mathrm{B} 2 \rightarrow \mathrm{B} 1$ & 2 & 1 & 1 & 1 & 2 \\
Verde & $\mathrm{B} 1 \rightarrow \mathrm{B} 2$ & 3 & 1 & 2 & 2 & 1 \\
Verde & $\mathrm{B} 2 \rightarrow \mathrm{B} 3$ & 3 & 1 & 1 & 2 & 1 \\
Vermelho & $\mathrm{B} 3 \rightarrow \mathrm{B} 2$ & 2 & 2 & 2 & 1 & 1 \\
Verde & $\mathrm{B} 3 \rightarrow \mathrm{B} 4$ & 2 & 1 & 2 & 2 & 1 \\
Vermelho & $\mathrm{B} 4 \rightarrow \mathrm{B} 3$ & 1 & 1 & 1 & 2 & 3 \\
Vermelho & $\mathrm{B} 5 \rightarrow \mathrm{B} 4$ & 3 & 1 & 1 & 1 & 2 \\
Verde & $\mathrm{B} 4 \rightarrow \mathrm{B} 5$ & 1 & 1 & 1 & 1 & 1 \\
Verde & $\mathrm{C} 1 \rightarrow \mathrm{C} 2$ & 2 & 2 & 2 & 1 & 2 \\
Verde & $\mathrm{C} 2 \rightarrow \mathrm{C} 3$ & 1 & 2 & 3 & 2 & 1 \\
Vermelho & $\mathrm{C} 2 \rightarrow \mathrm{C} 1$ & 1 & 1 & 2 & 1 & 2 \\
Vermelho & $\mathrm{C} 3 \rightarrow \mathrm{C} 2$ & 1 & 2 & 2 & 2 & 2 \\
Verde & $\mathrm{C} 3 \rightarrow \mathrm{C} 4$ & 1 & 1 & 3 & 1 & 1 \\
Vermelho & $\mathrm{C} 4 \rightarrow \mathrm{C} 3$ & 2 & 2 & 2 & 1 & 1 \\
Vermelho & $\mathrm{C} 5 \rightarrow \mathrm{C} 4$ & 1 & 2 & 2 & 1 & 1 \\
Verde & $\mathrm{C} 4 \rightarrow \mathrm{C} 5$ & 2 & 1 & 1 & 2 & 1 \\
\hline \multirow{2}{*}{ E Esthyyyyyy} & & & & &
\end{tabular}

* Estímulo condicional

Nota. 0 número é de 1 a 3 ou mais (>3).

Nas sondas (ver Tabela 4), de um modo geral todos os participantes apresentaram desempenho consistente com o treino na maioria das tentativas. Dois deles (JSO e PRS) responderam de acordo com o progra- mado em todas as tentativas. AMN, KVM e JVG responderam, respectivamente, de acordo com o programado em 22,21 e 23 das 24 tentativas. 
TABELA 4

Desempenho dos Participantes nas Sondas

\begin{tabular}{|c|c|c|c|c|c|c|}
\hline \multirow[b]{2}{*}{$\mathrm{sc}^{\mathrm{C}}$} & \multirow[b]{2}{*}{ Tipo de Tentativa } & \multicolumn{5}{|c|}{ Participantes } \\
\hline & & amn & jso & prs & kvm & jvg \\
\hline Verde & $\mathrm{A} 1 \rightarrow \mathrm{A} 2$ & * & * & * & * & * \\
\hline Vermelho & $\mathrm{A} 2 \rightarrow \mathrm{A} 1$ & * & * & * & ** & $* *$ \\
\hline Vermelho & $\mathrm{A} 3 \rightarrow \mathrm{A} 2$ & * & * & * & * & * \\
\hline Verde & $\mathrm{A} 2 \rightarrow \mathrm{A} 3$ & * & * & * & * & * \\
\hline Verde & $\mathrm{A} 3 \rightarrow \mathrm{A} 4$ & * & * & * & * & * \\
\hline Vermelho & $\mathrm{A} 4 \rightarrow \mathrm{A} 3$ & * & * & * & ** & * \\
\hline Vermelho & $\mathrm{A} 5 \rightarrow \mathrm{A} 4$ & * & * & * & * & * \\
\hline Verde & $\mathrm{A} 4 \rightarrow \mathrm{A} 5$ & * & * & * & * & * \\
\hline Vermelho & $\mathrm{B} 2 \rightarrow \mathrm{B} 1$ & * & * & * & ** & * \\
\hline Verde & $\mathrm{B} 1 \rightarrow \mathrm{B} 2$ & * & * & * & * & * \\
\hline Verde & $\mathrm{B} 2 \rightarrow \mathrm{B} 3$ & * & * & * & * & * \\
\hline Vermelho & $\mathrm{B} 3 \rightarrow \mathrm{B} 2$ & * & * & * & * & * \\
\hline Verde & $\mathrm{B} 3 \rightarrow \mathrm{B} 4$ & * & * & * & * & * \\
\hline Vermelho & $\mathrm{B} 4 \rightarrow \mathrm{B} 3$ & * & * & * & * & $*$ \\
\hline Vermelho & $\mathrm{B} 5 \rightarrow \mathrm{B} 4$ & * & * & * & * & * \\
\hline Verde & $\mathrm{B} 4 \rightarrow \mathrm{B} 5$ & * & * & * & * & * \\
\hline Verde & $\mathrm{C} 1 \rightarrow \mathrm{C} 2$ & * & * & * & * & * \\
\hline Verde & $\mathrm{C} 2 \rightarrow \mathrm{C} 3$ & * & * & * & * & $*$ \\
\hline Vermelho & $\mathrm{C} 2 \rightarrow \mathrm{C} 1$ & ** & * & * & * & * \\
\hline Vermelho & $\mathrm{C} 3 \rightarrow \mathrm{C} 2$ & * & * & * & * & * \\
\hline Verde & $\mathrm{C} 3 \rightarrow \mathrm{C} 4$ & * & * & * & * & * \\
\hline Vermelho & $\mathrm{C} 4 \rightarrow \mathrm{C} 3$ & * & * & * & * & * \\
\hline Vermelho & $\mathrm{C} 5 \rightarrow \mathrm{C} 4$ & * & * & * & * & $*$ \\
\hline Verde & $\mathrm{C} 4 \rightarrow \mathrm{C} 5$ & ** & * & * & * & * \\
\hline & & $22 / 24$ & $24 / 24$ & $24 / 24$ & $21 / 24$ & $23 / 24$ \\
\hline
\end{tabular}

Nota. Um asterisco significa que o participante continuou respondendo de acordo com as contingências a que foi exposto no treino, dois asteriscos significam resposta em desacordo com as contingências.

A Tabela 5 apresenta os resultados nos testes de transitividade. Três participantes (PRS, KVM e JVG) responderam corretamente, respectivamente, em 28 , 26 e 27 tentativas, do total de 36 tentativas programadas, na presença de ambas as cores. Os outros dois (AMN e JSO) apresentaram um desempenho diferente do programado na maioria das tentativas na presença de ambas as cores. Todos os participantes, à exceção de JSO, parecem ter desempenho melhor quando da aquisição das seqüências com o $2^{\circ}$ ou $3^{\circ}$ conjunto de estímulos em relação ao primeiro. Nenhum participante respondeu corretamente todas as tentativas quando expostos ao conjunto "A". Quando expostos ao conjunto "B" somente um participante (PRS) respondeu corretamente todas as tentativas. Na presença do conjunto "C" somente um participante (JVG) respondeu corretamente em todas as tentativas. 
TABELA 5

Desempenho dos Participantes nos Testes de Transitividade

\begin{tabular}{|c|c|c|c|c|c|c|}
\hline \multirow[b]{2}{*}{ SC } & \multirow[b]{2}{*}{$\begin{array}{l}\text { Tipo de } \\
\text { Tentativa }\end{array}$} & \multicolumn{5}{|c|}{ Participantes } \\
\hline & & amn & jso & prs & kvm & jvg \\
\hline Verde & $\mathrm{A} 1 \rightarrow \mathrm{A} 3$ & * & $\star \star$ & ** & * & ** \\
\hline Verde & $\mathrm{A} 1 \rightarrow \mathrm{A} 5$ & * & ** & * & * & * \\
\hline Vermelho & $\mathrm{A} 4 \rightarrow \mathrm{A} 1$ & * & * & ** & ** & * \\
\hline Verde & $\mathrm{A} 2 \rightarrow \mathrm{A} 5$ & * & * & ** & ** & * \\
\hline Vermelho & $\mathrm{A} 3 \rightarrow \mathrm{A} 1$ & ** & * & * & * & * \\
\hline Verde & $\mathrm{A} 2 \rightarrow \mathrm{A} 4$ & * & * & ** & * & * \\
\hline Vermelho & $\mathrm{A} 5 \rightarrow \mathrm{A} 3$ & * & ** & * & * & ** \\
\hline Vermelho & $\mathrm{A} 4 \rightarrow \mathrm{A} 2$ & ** & * & ** & ** & * \\
\hline Verde & $\mathrm{A} 1 \rightarrow \mathrm{A} 4$ & ** & * & * & ** & * \\
\hline Verde & $\mathrm{A} 3 \rightarrow \mathrm{A} 5$ & ** & ** & * & * & ** \\
\hline Vermelho & $\mathrm{A} 5 \rightarrow \mathrm{A} 2$ & ** & * & * & * & ** \\
\hline \multirow{2}{*}{ Vermelho } & $\mathrm{A} 5 \rightarrow \mathrm{A} 1$ & ** & * & * & * & ** \\
\hline & & $6 / 12$ & $8 / 12$ & $7 / 12$ & $8 / 12$ & $7 / 12$ \\
\hline Verde & $\mathrm{B} 2 \rightarrow \mathrm{B} 4$ & * & * & * & ** & * \\
\hline Vermelho & $\mathrm{B} 3 \rightarrow \mathrm{B} 1$ & ** & * & * & ** & ** \\
\hline Verde & $\mathrm{B} 2 \rightarrow \mathrm{B} 5$ & * & * & * & * & * \\
\hline Vermelho & $\mathrm{B} 4 \rightarrow \mathrm{B} 1$ & * & * & * & * & * \\
\hline Vermelho & $\mathrm{B} 5 \rightarrow \mathrm{B} 2$ & ** & ** & * & ** & * \\
\hline Verde & $\mathrm{B} 1 \rightarrow \mathrm{B} 5$ & * & ** & * & * & * \\
\hline Verde & B3 $\rightarrow$ B5 & ** & ** & * & * & * \\
\hline Vermelho & $\mathrm{B} 5 \rightarrow \mathrm{B} 1$ & ** & * & * & * & ** \\
\hline Vermelho & $\mathrm{B} 4 \rightarrow \mathrm{B} 2$ & ** & ** & * & * & * \\
\hline Verde & $\mathrm{B} 1 \rightarrow \mathrm{B} 3$ & * & * & * & ** & ** \\
\hline Vermelho & $\mathrm{B} 5 \rightarrow \mathrm{B} 3$ & * & * & * & * & ** \\
\hline \multirow[t]{2}{*}{ Verde } & $\mathrm{B} 1 \rightarrow \mathrm{B} 4$ & * & * & * & ** & * \\
\hline & & $7 / 12$ & $8 / 12$ & $12 / 12$ & $7 / 12$ & $8 / 12$ \\
\hline Vermelho & $\mathrm{C} 4 \rightarrow \mathrm{C} 1$ & ** & * & * & * & * \\
\hline Verde & $\mathrm{C} 2 \rightarrow \mathrm{C} 5$ & * & ** & * & * & * \\
\hline Verde & $\mathrm{C} 1 \rightarrow \mathrm{C} 5$ & * & * & ** & ** & * \\
\hline Vermelho & $\mathrm{C} 3 \rightarrow \mathrm{C} 1$ & ** & * & * & * & * \\
\hline Vermelho & $\mathrm{C} 5 \rightarrow \mathrm{C} 2$ & * & ** & * & * & * \\
\hline Verde & $\mathrm{C} 1 \rightarrow \mathrm{C} 4$ & ** & * & * & * & * \\
\hline Verde & $\mathrm{C} 1 \rightarrow \mathrm{C} 3$ & ** & ** & * & * & * \\
\hline Vermelho & $\mathrm{C} 4 \rightarrow \mathrm{C} 2$ & * & * & * & * & * \\
\hline Verde & $\mathrm{C} 3 \rightarrow \mathrm{C} 5$ & * & ** & * & * & * \\
\hline Vermelho & $\mathrm{C} 5 \rightarrow \mathrm{C} 1$ & ** & * & ** & * & * \\
\hline Vermelho & $\mathrm{C} 5 \rightarrow \mathrm{C} 3$ & * & ** & * & * & * \\
\hline \multirow[t]{3}{*}{ Verde } & $\mathrm{C} 2 \rightarrow \mathrm{C} 4$ & * & * & ** & * & * \\
\hline & & $7 / 12$ & $7 / 12$ & 9/12 & $11 / 12$ & $12 / 12$ \\
\hline & & $20 / 36$ & $23 / 36$ & $28 / 36$ & $26 / 36$ & $27 / 36$ \\
\hline
\end{tabular}

Nota. Um asterisco significa que o participante respondeu de modo que permite inferir a propriedade transitiva, ao passo que dois asteriscos significam o contrário.

Na revisão de linha de base (ver Tabela 6), todos os participantes alcançaram o critério de acerto, embora precisassem de duas exposições em várias tentativas nos três conjuntos de estímulos A, B e C. Um participante (JVG) precisou de duas exposições em três seqüências $(\mathrm{A} 2 \rightarrow \mathrm{A} 1 ; \mathrm{A} 3 \rightarrow \mathrm{A} 2 ; \mathrm{B} 5 \rightarrow \mathrm{B} 4)$ na presença da cor vermelha e respondeu corretamente na primeira tentativa em 21 das 24 tentativas programadas. Dois participantes (JSO e KVM) responderam corretamente na primeira tentativa, respectivamente, em 16 e 18 tentativas na presença de ambas as cores, de um total de 24 tentativas. Os outros dois participantes precisaram de duas exposições na maioria das tentativas. 
TABELA 6

Desempenho dos Participantes na Revisão de Linha de Base

\begin{tabular}{|c|c|c|c|c|c|c|}
\hline \multirow[b]{2}{*}{ Sc } & \multirow[b]{2}{*}{ Tipo de Tentativa } & \multicolumn{5}{|c|}{ Participantes } \\
\hline & & AMN & JSO & PRS & KVM & JVG \\
\hline Verde & $\mathrm{A} 3 \rightarrow \mathrm{A} 4$ & ** & * & * & * & * \\
\hline Vermelho & $\mathrm{A} 2 \rightarrow \mathrm{A} 1$ & * & * & ** & * & $* *$ \\
\hline Vermelho & $\mathrm{A} 3 \rightarrow \mathrm{A} 2$ & ** & * & ** & * & $* *$ \\
\hline Verde & $\mathrm{A} 4 \rightarrow \mathrm{A} 5$ & * & * & ** & * & * \\
\hline Verde & $\mathrm{A} 1 \rightarrow \mathrm{A} 2$ & ** & * & * & * & * \\
\hline Vermelho & $\mathrm{A} 4 \rightarrow \mathrm{A} 3$ & * & ** & ** & * & * \\
\hline Vermelho & $\mathrm{A} 5 \rightarrow \mathrm{A} 4$ & * & ** & ** & * & * \\
\hline Verde & $\mathrm{A} 2 \rightarrow \mathrm{A} 3$ & * & * & ** & ** & * \\
\hline Vermelho & $\mathrm{B} 4 \rightarrow \mathrm{B} 3$ & $* *$ & * & ** & * & * \\
\hline Verde & $\mathrm{B} 4 \rightarrow \mathrm{B} 5$ & ** & * & ** & * & * \\
\hline Verde & $\mathrm{B} 2 \rightarrow \mathrm{B} 3$ & * & ** & ** & * & * \\
\hline Vermelho & $\mathrm{B} 5 \rightarrow \mathrm{B} 4$ & * & ** & ** & * & $* *$ \\
\hline Vermelho & $\mathrm{B} 2 \rightarrow \mathrm{B} 1$ & ** & * & * & ** & * \\
\hline Verde & $\mathrm{B} 3 \rightarrow \mathrm{B} 4$ & * & ** & * & ** & * \\
\hline Vermelho & $\mathrm{B} 3 \rightarrow \mathrm{B} 2$ & * & * & ** & ** & * \\
\hline Verde & $\mathrm{B} 1 \rightarrow \mathrm{B} 2$ & ** & * & * & * & * \\
\hline Verde & $\mathrm{C} 1 \rightarrow \mathrm{C} 2$ & ** & * & * & ** & * \\
\hline Vermelho & $\mathrm{C} 5 \rightarrow \mathrm{C} 4$ & ** & ** & ** & * & * \\
\hline Vermelho & $\mathrm{C} 4 \rightarrow \mathrm{C} 3$ & ** & * & ** & * & * \\
\hline Verde & $\mathrm{C} 4 \rightarrow \mathrm{C} 5$ & * & * & * & ** & * \\
\hline Vermelho & $\mathrm{C} 3 \rightarrow \mathrm{C} 2$ & * & ** & * & * & * \\
\hline Verde & $\mathrm{C} 3 \rightarrow \mathrm{C} 4$ & ** & ** & * & * & * \\
\hline Verde & $\mathrm{C} 2 \rightarrow \mathrm{C} 3$ & * & * & ** & * & * \\
\hline Vermelho & $\mathrm{C} 2 \rightarrow \mathrm{C} 1$ & * & * & * & * & * \\
\hline & & $13 / 24$ & $16 / 24$ & $10 / 24$ & $18 / 24$ & $21 / 24$ \\
\hline
\end{tabular}

Embora os melhores desempenhos na aquisição e inferência transitiva sejam para PRS, KVM e JVG, esse desempenho se mantém apenas para KVM e JVG na revisão da linha de base.

A Tabela 7 apresenta os resultados nos testes de conectividade e formação de classes ordinais com elementos de conjuntos distintos. Do total de 48 tentativas programadas, os participantes AMN, JSO, PRS, KVM e JVG responderam de acordo com o programado, respectivamente, em $31,42,35,43$ e 38 tentativas na presença de ambas as cores. Porém, três participantes (PRS, KVM e JVG) responderam corretamente todas as tentativas da relação A/B. Quando expostos à relação $\mathrm{B} / \mathrm{A}$ nenhum participante respondeu corretamente. Quando expostos à relação $\mathrm{A} / \mathrm{C}$, três participantes (JSO, KVM e JVG) responderam corretamente em todas as tentativas. $\mathrm{O}$ participante JSO foi o único a responder corretamente em todas as tentativas da relação C/A. Nenhum participante respondeu corretamente a relação $\mathrm{C} / \mathrm{B}$ e dois participantes (JSO e KVM) responderam corretamente em todas as tentativas da relação $\mathrm{B} / \mathrm{C}$. 
TABELA 7

Desempenho dos Participantes nos Testes de Conectividade e Formação de Classes com Elementos de Conjuntos Distintos

\begin{tabular}{|c|c|c|c|c|c|c|}
\hline \multirow[b]{2}{*}{ Sc } & \multirow[b]{2}{*}{ Tipo de Tentativa } & \multicolumn{5}{|c|}{ Participantes } \\
\hline & & amn & jso & prs & kvm & jvg \\
\hline Verde & $\mathrm{A} 1 \rightarrow \mathrm{B} 2$ & * & * & $*$ & * & * \\
\hline Vermelho & $\mathrm{A} 2 \rightarrow \mathrm{B} 1$ & * & * & * & * & * \\
\hline Verde & $\mathrm{A} 2 \rightarrow \mathrm{B} 3$ & * & * & * & * & * \\
\hline Vermelho & $\mathrm{A} 3 \rightarrow \mathrm{B} 2$ & * & * & * & * & * \\
\hline Verde & $\mathrm{A} 3 \rightarrow \mathrm{B} 4$ & ** & $* *$ & * & * & * \\
\hline Vermelho & $\mathrm{A} 4 \rightarrow \mathrm{B} 3$ & ** & * & * & * & * \\
\hline Verde & $\mathrm{A} 4 \rightarrow \mathrm{B} 5$ & ** & * & * & * & * \\
\hline \multirow[t]{2}{*}{ Vermelho } & $\mathrm{A} 5 \rightarrow \mathrm{B} 4$ & * & $* *$ & * & * & * \\
\hline & & $5 / 8$ & $6 / 8$ & $8 / 8$ & $8 / 8$ & $8 / 8$ \\
\hline Verde & $\mathrm{B} 1 \rightarrow \mathrm{A} 2$ & * & $* *$ & $* *$ & ** & * \\
\hline Vermelho & $\mathrm{B} 2 \rightarrow \mathrm{A} 1$ & ** & * & $* *$ & * & * \\
\hline Verde & $\mathrm{B} 2 \rightarrow \mathrm{A} 3$ & $* *$ & * & * & * & * \\
\hline Vermelho & $\mathrm{B} 3 \rightarrow \mathrm{A} 2$ & * & * & $* *$ & * & * \\
\hline Verde & $\mathrm{B} 3 \rightarrow \mathrm{A} 4$ & ** & * & $* *$ & * & ** \\
\hline Vermelho & $\mathrm{B} 4 \rightarrow \mathrm{A} 3$ & * & * & * & ** & * \\
\hline Verde & $\mathrm{B} 4 \rightarrow \mathrm{A} 5$ & * & * & $* *$ & * & ** \\
\hline \multirow[t]{2}{*}{ Vermelho } & $\mathrm{B} 5 \rightarrow \mathrm{A} 4$ & * & * & * & * & ** \\
\hline & & $5 / 8$ & $7 / 8$ & $3 / 8$ & $6 / 8$ & $5 / 8$ \\
\hline Verde & $\mathrm{A} 2 \rightarrow \mathrm{C} 1$ & ** & * & * & * & * \\
\hline Vermelho & $\mathrm{A} 3 \rightarrow \mathrm{C} 4$ & * & * & * & * & * \\
\hline Verde & $\mathrm{A} 2 \rightarrow \mathrm{C} 3$ & ** & * & * & * & * \\
\hline Vermelho & $\mathrm{A} 4 \rightarrow \mathrm{C} 3$ & $* *$ & * & * & * & * \\
\hline Verde & $\mathrm{A} 1 \rightarrow \mathrm{C} 2$ & * & * & $*$ & * & * \\
\hline Vermelho & $\mathrm{A} 3 \rightarrow \mathrm{C} 2$ & $* *$ & * & * & * & * \\
\hline Verde & $\mathrm{A} 5 \rightarrow \mathrm{C} 4$ & $* *$ & * & $* *$ & * & * \\
\hline \multirow[t]{2}{*}{ Vermelho } & $\mathrm{A} 4 \rightarrow \mathrm{C} 5$ & ** & * & * & * & * \\
\hline & & $2 / 8$ & $8 / 8$ & $7 / 8$ & $8 / 8$ & $8 / 8$ \\
\hline Verde & $\mathrm{C} 1 \rightarrow \mathrm{A} 2$ & * & $* *$ & * & ** & ** \\
\hline Vermelho & $\mathrm{C} 3 \rightarrow \mathrm{A} 2$ & ** & * & * & * & * \\
\hline Verde & $\mathrm{C} 3 \rightarrow \mathrm{A} 4$ & * & * & * & * & * \\
\hline Vermelho & $\mathrm{C} 5 \rightarrow \mathrm{A} 4$ & * & * & $* *$ & * & ** \\
\hline Verde & $\mathrm{C} 2 \rightarrow \mathrm{A} 1$ & * & * & * & * & * \\
\hline Vermelho & $\mathrm{C} 2 \rightarrow \mathrm{A} 3$ & * & * & $* *$ & * & * \\
\hline Verde & $\mathrm{C} 4 \rightarrow \mathrm{A} 5$ & * & * & $*$ & * & * \\
\hline \multirow[t]{2}{*}{ Vermelho } & $\mathrm{C} 4 \rightarrow \mathrm{A} 3$ & * & * & $*$ & * & * \\
\hline & & $7 / 8$ & $8 / 8$ & $6 / 8$ & $7 / 8$ & $6 / 8$ \\
\hline
\end{tabular}




\begin{tabular}{|c|c|c|c|c|c|c|}
\hline Vermelho & $\mathrm{C} 4 \rightarrow \mathrm{B} 3$ & * & * & * & $* *$ & $* *$ \\
\hline Verde & $\mathrm{C} 3 \rightarrow \mathrm{B} 4$ & * & * & * & * & $* *$ \\
\hline Vermelho & $\mathrm{C} 1 \rightarrow \mathrm{B} 2$ & * & * & ** & * & * \\
\hline Verde & $\mathrm{C} 5 \rightarrow \mathrm{B} 4$ & * & * & $* *$ & * & $* *$ \\
\hline Vermelho & $\mathrm{C} 2 \rightarrow \mathrm{B} 3$ & $* *$ & * & $* *$ & * & * \\
\hline Verde & $\mathrm{C} 2 \rightarrow \mathrm{B} 1$ & * & * & * & * & * \\
\hline Vermelho & $\mathrm{C} 3 \rightarrow \mathrm{B} 2$ & * & $* *$ & * & $* *$ & * \\
\hline \multirow[t]{2}{*}{ Verde } & $\mathrm{C} 4 \rightarrow \mathrm{B} 5$ & * & $* *$ & * & * & * \\
\hline & & $7 / 8$ & $6 / 8$ & $5 / 8$ & $6 / 8$ & $5 / 8$ \\
\hline Verde & $\mathrm{B} 1 \rightarrow \mathrm{C} 2$ & * & * & $* *$ & * & $*$ \\
\hline Vermelho & $\mathrm{B} 3 \rightarrow \mathrm{C} 4$ & $* *$ & * & * & * & $*$ \\
\hline Verde & $\mathrm{B} 5 \rightarrow \mathrm{C} 4$ & * & * & * & * & * \\
\hline Vermelho & $\mathrm{B} 3 \rightarrow \mathrm{C} 2$ & ** & * & * & * & * \\
\hline Verde & $\mathrm{B} 4 \rightarrow \mathrm{C} 5$ & $* *$ & * & * & * & $* *$ \\
\hline Vermelho & $\mathrm{B} 4 \rightarrow \mathrm{C} 3$ & * & * & $* *$ & * & $* *$ \\
\hline Verde & $\mathrm{B} 2 \rightarrow \mathrm{C} 1$ & * & * & * & * & $*$ \\
\hline \multirow[t]{3}{*}{ Vermelho } & $\mathrm{B} 2 \rightarrow \mathrm{C} 3$ & * & * & * & * & * \\
\hline & & $5 / 8$ & $8 / 8$ & $6 / 8$ & $8 / 8$ & $6 / 8$ \\
\hline & & $31 / 48$ & $42 / 48$ & $35 / 48$ & $43 / 48$ & $38 / 48$ \\
\hline
\end{tabular}

Nota. Um asterisco significa que o participante respondeu de modo que permite inferir a propriedade, ao passo que dois asteriscos significam o contrário.

Nos testes de transferência de função ordinal (ver Tabela 8), do total de oito tentativas programadas, um participante $(\mathrm{AMN})$ respondeu corretamente em seis tentativas na primeira oportunidade. Dois participan- tes (JSO e KVM) responderam de acordo com o programado em cinco das oito tentativas previstas. Outros dois (PRS e JVG) responderam de acordo com o programado em metade das tentativas previstas.

TABELA 8

Desempenho dos Participantes nos Testes de Transferência de Função

\begin{tabular}{ccccccc}
\hline & & \multicolumn{5}{c}{ Participantes } \\
\cline { 3 - 7 } Sc & Tipo de Tentativa & amn & jso & prs & kvm & jvg \\
\hline Verde & D1 $\rightarrow$ D2 & $*$ & $*$ & $*$ & $* *$ & $*$ \\
Vermelho & D5 $\rightarrow$ D4 & $*$ & $* *$ & $*$ & $* *$ & $* *$ \\
Vermelho & D3 $\rightarrow$ D2 & $*$ & $*$ & $* *$ & $*$ & $*$ \\
Verde & D3 $\rightarrow$ D4 & $*$ & $*$ & $*$ & $*$ & $* *$ \\
Vermelho & D2 $\rightarrow$ D1 & $* *$ & $*$ & $*$ & $* *$ & $* *$ \\
Verde & D4 $\rightarrow$ D5 & $*$ & $* *$ & $* *$ & $*$ & $*$ \\
Verde & D2 $\rightarrow$ D3 & $*$ & $*$ & $* *$ & $*$ & $* *$ \\
Vermelho & D4 $\rightarrow$ D3 & $* *$ & $* *$ & $* *$ & $*$ & $*$ \\
& & $6 / 8$ & $5 / 8$ & $4 / 8$ & $5 / 8$ & $4 / 8$ \\
\hline
\end{tabular}

Nota. Um asterisco indica a transferência e dois asteriscos indicam o contrário.

Nos testes de generalização, dois participantes (JSO e JVG) responderam corretamente à contagem dos objetos isoladamente (borrachas, copos e caixas de fósforos) ou em conjunto (ver Tabela 9) em todas 
as tentativas. Os demais participantes (AMN, PRS e $\mathrm{KVM}$ ) responderam corretamente, respectivamente, em 10,16 e 14 tentativas de um total de 20 programadas.

\section{TABELA 9}

Desempenho dos Participantes nos Testes de Generalização

\begin{tabular}{|c|c|c|c|c|c|c|}
\hline \multirow[b]{2}{*}{ Objeto } & \multirow[b]{2}{*}{ Quantidade } & \multicolumn{5}{|c|}{ Participantes } \\
\hline & & AMN & JSO & PRS & KVM & JVG \\
\hline \multirow{5}{*}{ Borrachas } & 1 & - & * & * & - & * \\
\hline & 2 & - & * & - & * & * \\
\hline & 3 & - & * & * & * & * \\
\hline & 4 & * & * & * & * & * \\
\hline & 5 & - & * & - & * & * \\
\hline \multirow{5}{*}{ Caixas de fósforos } & 1 & * & * & * & - & * \\
\hline & 2 & * & * & * & * & * \\
\hline & 3 & * & * & * & - & * \\
\hline & 4 & * & * & * & - & * \\
\hline & 5 & - & * & - & * & * \\
\hline \multirow{5}{*}{ Copos } & 1 & - & * & * & - & * \\
\hline & 2 & - & * & - & - & * \\
\hline & 3 & * & * & * & * & * \\
\hline & 4 & * & * & * & * & * \\
\hline & 5 & - & * & * & * & * \\
\hline 1 borracha & 1 & - & * & * & * & * \\
\hline 1 copo e 1 borracha & 2 & * & * & * & * & * \\
\hline 1 copo, 1 caixa e 1 borracha & 3 & * & * & * & * & * \\
\hline 2 copos, 1 caixa e 1 borracha & 4 & * & * & * & * & * \\
\hline 3 caixas e 2 borrachas & 5 & - & * & * & * & * \\
\hline
\end{tabular}

Nota. $\mathrm{O}$ asterisco $\left(^{*}\right)$ indica que o participante respondeu ao número correspondente e o hífen (-) indica resposta a outro número.

\section{DISCUSSÃO}

Todos os participantes alcançaram o critério de acerto no ensino e responderam corretamente a maioria das tentativas nas sondas aplicadas imediatamente após cada seqüência programada, na presença das cores verde e vermelha. Nas fases de sonda, inseridas entre as tentativas de ensino, o desempenho dos participantes foi consistente com o ensino, o que corrobora a proposta teórica da coerência da topografia de controle de estímulos (McIlvane, Serna, Dube \& Stromer, 2000), que exige a necessidade de programar testes inseridos entre tentativas de linha de base para que os testes não produzam controle por variáveis não planejadas. Segundo esses autores, mesmo procedimentos cuidadosos podem favorecer o desenvolvimento de mais de uma topografia de controle de estímulos, uma vez que relações de controle diferentes para uma mesma resposta podem levar a um mesmo resultado.
Então, uma topografia de controle de estímulos não planejada não precisa necessariamente concorrer com a desejada.

Três participantes responderam a maioria das tentativas acima de $66 \%$ do total de tentativas programadas. Portanto, os resultados desses três participantes são positivos na maior parte dos testes conduzidos e o resultado sai da zona de acaso. Os outros dois participantes responderam abaixo desse valor, indicando uma variabilidade inter-sujeitos. Entretanto, esse efeito parece ter dissipado rapidamente, uma vez que, para os dois participantes isso não foi condição suficiente para impedir que eles respondessem às novas seqüências envolvendo a permutação de estímulos com seqüências diferentes nos testes de conectividade. Green e Saunders (1998) apontam que a emergência de relações equivalentes nem sempre ocorre na primeira tentativa, mas gradualmente. 
Em seguida, na revisão de linha de base, todos os participantes alcançaram o critério, mas precisaram de duas exposições na maioria das tentativas para avançar para os testes de conectividade. Nestes testes, todos os participantes responderam na primeira oportunidade a maioria das tentativas programadas, ou seja, acima de $50 \%$ do total de tentativas previstas no testes, verificando-se, assim, a emergência de relações ordinais, sob controle condicional, com a utilização do procedimento de ensino por sobreposição de estímulos.

Os resultados replicam aqueles apresentados na literatura (Holcomb e cols., 1997; Stromer \& Mackay, Experimento 2, 1993; Souza, Assis \& Magalhães, 2005; Verdu, de Souza \& Lopes Jr., 2006) envolvendo o ensino por sobreposição de estímulos e amplia os estudos sob contingências mais complexas, sugerindo a produção de novas seqüências, sob controle condicional, sem qualquer ensino adicional em crianças surdas.

Segundo autores como Holcomb e cols. (1997), o procedimento de sobreposição de estímulos envolvendo fading (esvanecimento) foi eficaz se comparado aos de tentativa-e-erro, trazendo novos achados para a área de inferência transitiva. Os procedimentos adotados nesses estudos (Holcomb e cols., 1997; Stromer \& Mackay, Experimento 2, 1993) têm em comum o uso do fading de diferentes intensidades servindo como estímulo facilitador durante o ensino de pares de estímulos não-adjacentes.

No estudo aqui relatado, houve emergência de relações entre estímulos funcionalmente equivalentes entre diferentes seqüências de estímulos, sob controle condicional, inferindo-se as propriedades de transitividade e conectividade, sugerindo a formação de relações ordinais em que os estímulos ocupavam a mesma posição em diferentes seqüências (cf. Green e cols., 1993).

Esses dados mostram o pioneirismo do estudo e a importância da utilização do procedimento de ensino por sobreposição de estímulos na emergência de relações ordinais com pares de estímulos não-adjacentes, sob controle condicional, em crianças com surdez congênita. Entretanto, esses resultados não tão consistentes parecem corroborar com as palavras de Green (1990) que enfatiza a relação do ensino na modalidade auditiva-visual para aprendizagem de comportamentos complexos.

A demonstração do controle condicional sobre o responder seqüencial é uma importante contribuição científica com implicações no ensino, devido às habilidades exigidas em repertórios de leitura, escrita ou matemática, nos quais a ordem é uma variável relevante na execução dessas tarefas acadêmicas.

Os testes de transferência de funções ordinais aplicados também apresentam resultados promissores, uma vez que todos os participantes responderam consistentemente com a linha de base, embora dois participantes tenham respondido a metade das tentativas.

Testes de generalização também foram aplicados para avaliar a generalidade dos dados, com estímulos do ambiente natural. Dois participantes (JSO e JVG) foram capazes de responder na primeira tentativa à propriedade de numerosidade. Dois responderam a maioria das tentativas e um participante respondeu a metade das tentativas programadas.

Uma extensão deste estudo no futuro deve verificar sob quais condições pode ser obtido o responder seqüencial sob controle condicional com mais de dois estímulos, e verificar se a ordem em que a seqüência é ensinada (por exemplo, linear ou aleatória) na presença de estímulos diferentes interfere na aprendizagem direta e emergente nesta população.

\section{REFERÊNCIAS}

Assis, G. J. A. (1987). Comportamento de ordenação: uma análise experimental de algumas variáveis. Psicologia: Teoria e Pesquisa, 3, 224-238.

Assis, G. J. A., \& Costa, L. C. A. (2004). Emergência de relações ordinais em crianças. Interação em Psicologia, 8(2), 199-216.

Carmo, J. (2002). Definições operacionais de habilidades matemáticas elementares. Em H. Guilhardi, M. B. B. Madi, P. P. Queiroz \& C. M. C. Scoz, (Eds.), Sobre comportamento e cognição: Vol. 9. Contribuições para a construção da teoria do comportamento (pp.181-191). Santo André: ESETec.

Galy, E., Camps, J. F., \& Melan, C. (2003). Sequence class formation following learning of short sequences. The Psychological Record, 53, 635-645.

Green, G. (1990). Differences in development of visual and auditory-visual equivalence relations. American Journal on Mental Retardation, 95(3), 260-270.

Green, G. (1993). Stimulus control technology for teaching number-quantity equivalences. Em Victoria Autistic Children's and Adults Association (Org.), Proceedings of the 1992 Conference of the National Association for Autism (Australia) (pp. 51-63). Melbourne, Austrália.

Green, G., \& Saunders, R. R. (1998). Stimulus equivalence. Em K. Lattal, \& M. Perone, (Orgs.) Handbook of research methods in human operant behavior (pp. 229-262). New York: Plenum Press.

Green, G., Stromer, R., \& Mackay, H. (1993). Relational learning in stimulus sequences. The Psychological Record, 43, 599-616.

Holcomb, W. L., Stromer, R., \& Mackay, H. A. (1997). Transitivity and emergent sequence performances in young children. Journal of Experimental Child Psychology, 65, 96-124. 
Lima, M. P., \& Assis, G. J. A. (2003). Emergência de classes seqüenciais após treino com pareamento consistente. Psicologia: Teoria e Pesquisa, 19(1), 75-84.

Mackay, H. A., \& Brown, S. M. (1971). Teaching serial position sequences to monkey with a delayed matching-to-sample procedure. Journal of the Experimental Analysis of Behavior, 15, 335-345.

Mackay, H. A., Kotlarchyk, B. J., \& Stromer, R. (1997). Stimulus classes, stimulus sequences, and generative behavior. Em D. Baer \& E. M. Pinkston (Orgs.), Environment and behavior (pp. 124-137). Boulder, CO: WestviewPress.

McIlvane, W. J., Serna, R., Dube, W. V., \& Stromer, R. (2000). Stimulus control topography coherence and stimulus equivalence: reconciling test outcomes with theory. Em J. C. Leslie \& D. E. Blackman (Eds.), Experimental and applied analysis of human behavior (pp. 85-110). Reno, NV: Context Press.

Ribeiro, M. P. L., Assis G. J. A., \& Enumo, S. R. F. (2005). Controle do comportamento por relações ordinais: questões conceituais e metodológicas. Em E. B. Borloti, S. R. F. Enumo e M. L. P. Ribeiro (Orgs), Análise do comportamento: Teorias e práticas (p. 117-132). Santo André: ESETec.

Sampaio, M. E. C., \& Assis, G. J. A. (2005). Equivalência de estímulos seqüenciais em portadores de necessidades educacionais especiais. Acta Comportamentalia, 13(2), 111-143.

Sidman, M., \& Rosemberger, P. B. (1967). Several methods for teaching serial position sequences to monkeys. Journal of the Experimental Analysis of Behavior, 10, 467-478.

Sigurdardottir, Z. G., Green, G., \& Saunders, R. R. (1990). Equivalence classes generated by sequence training. Journal of the Experimental Analysis of Behavior, 53, 47-63.

Souza, R. D. C., Assis, G. J. A., \& Magalhães, P. G. S. (2005). Equivalência numérica em crianças surdas. Temas em Psicologia, 22(2), 113-127.

Stromer, R., \& Mackay, H. A. (1993). Human sequential behavior: relations among stimuli, class formation, and derived sequences. The Psychological Record, 43, 107-131.

Verdu, A. C. M. A., de Souza, D. G., \& Lopes Jr., J. (2006). Formação de classes ordinais após a aprendizagem de seqüenciais independentes. Estudos de Psicologia, 11(1), 87-99.

Recebido: $31 / 07 / 2007$ Última revisão: 04/05/2008 Aceite final: 25/05/2008

\section{Notas}

Agradecemos ao programador Márcio Braga dos Santos pelo apoio técnico na confecção do software de coleta de dados comportamentais.

${ }^{1}$ Green e cols. (1993) adotam um código alfanumérico, no qual letras maiúsculas são usadas para representar classes de estímulos e numerais seus elementos. O símbolo " $\rightarrow$ “indica qual elemento precede o outro. A mesma notação será usada aqui.

\section{Sobre os autores:}

Ruth Daisy Capistrano de Souza: Doutora pelo Programa de Pós-Graduação em Teoria e Pesquisa do Comportamento, Universidade Federal do Pará. Endereço eletrônico: ruthcapistrano@yahoo.com.br.

Grauben José Alves de Assis: Doutor em Psicologia Experimental pela Universidade de São Paulo. Docente da Faculdade de Psicologia e do Programa de Pós-Graduação em Teoria e Pesquisa do Comportamento, Universidade Federal do Pará. Bolsista de Produtividade em Pesquisa do CNPq.

Priscila Giselli Silva Magalhães: Mestranda no Programa de Pós-Graduação em Teoria e Pesquisa do Comportamento, Universidade Federal do Pará.

Paulo Sérgio Teixeira Prado: Doutor em Psicologia Experimental pela Universidade de São Paulo. Docente na Universidade Estadual Paulista "Júlio de Mesquita Filho", Campus de Marília. Endereço eletrônico: prado01@flash.tv.br.

Endereço para correspondência: Grauben José Alves de Assis. Trav. Castelo Branco, 1.923/301 - Bairro do Guamá, 66.063.420 Belém-Pará. Endereço eletrônico: grauben@pesquisador.cnpq.br. 